\title{
CLINICAL AND ELECTROCARDIOGRAPHIC PROFILE OF PATIENTS PRESENTING WITH ACUTE CORONARY SYNDROME IN EMERGENCY DEPARTMENT IN A TERTIARY CARE HOSPITAL.
}

1. MBBS, M.Phil (Biochemistry), Assistant Professor Biochemistry Wah Medical College.

2. MBBS, M.Phil (Biochemistry)

Professor \& Allied Medical Profession, HOD Biochemistry Sahara Medical College Narowal.

3. MBBS, FCPS (Clinical Path)

Professor of Pathology

Wah Medical College.

4. MBBS, FCPS (Med)

Assistant Professor Medicine Wah Medical College.

5. M.Phil

Assistant Professor Rehabilitation Islamabad Medical and Dental College, Islamabad.

Correspondence Address:

Dr. Naveed Arshad

Department of Rehabilitation Islamabad Medical and Dental

College, Islamabad,

Main Murree Road, Sattran Meel,

Bhara Kahu, Islamabad.

oliajann@gmail.com

Article received on:

23/12/2019

Accepted for publication:

21/02/2020

\section{Zahid Mahmood ${ }^{1}$, Tariq Feroze Khawaja ${ }^{2}$, Anjum Iqbal ${ }^{3}$, Abdul Rashid Khan ${ }^{4}$, Naveed Arshad}

ABSTRACT... Objectives: To assess the clinical characteristics and diagnosis of ACS for timely, management and further prevention from coronary events. Study Design: Cross sectional study. Setting: Emergency Department (ED) of Punjab Institute of Cardiology (PIC) Lahore. Period: November 2017 to January 2018. Material \& Methods: Included 170 diagnosed patients of ACS of both sexes presenting within approximately four hours of symptoms. Results: A total of 170 patients with established diagnosis of ACS were included. The mean age of the patients was 56 years. There was a very strong male predominance $(81.76 \%)$. The major modifiable risk factors were hypertension (54.71\%) and smoking (35.88\%), followed by hyperlipidemia (35.29\%) and diabetes (32.35\%). A previous history of IHD in patients and family history of IHD were equally present $(37.65 \%)$. A large proportion of patients belonged to middle class $(54.12 \%)$ and lower (41.18\%). Typical pain chest pain was present in $90 \%$ and majority of patients enrolled in this study had a diagnosis of myocardial infarction $77.05 \%$ and the remainder had unstable angina. We found a higher percentage of ST elevation MI in the patients with $\mathrm{MI}(84.73 \%)$, majority had anterior AMI. Of all patients, $1.77 \%$ expired during hospital stay. Conclusion: The majority of ACS patients in our country are male with STEMI. The major risk factors are hypertension, family history of IHD, smoking, hyperlipidemia and diabetes. $90 \%$ patients present with typical chest pain while remaining $10 \%$ atypical symptoms which must be of prime consideration to assess ACS.

Key words: $\quad$ Acute Coronary Syndrome, Myocardial Infarction, Risk Factors

Article Citation: Mahmood Z, Khawaja TF, Iqbal A, Khan AR, Arshad N. Clinical and electrocardiographic profile of patients presenting with acute coronary syndrome in emergency department in a Tertiary Care Hospital. Professional Med J 2020; 27(8):1669-1674. DOI: 10.29309/TPMJ/2020.27.08.4437

\section{INTRODUCTION}

According to recent statistics of World Health Organization, the most common cause of death in the world is coronary heart disease (CHD) accounting almost 9.5 million deaths in year 2016. ${ }^{1}$ The rates of main cardiovascular events and death have been found to be significantly superior in low-income countries compared to high-income countries, inspite of the reality that these countries have the lowest risk-factor. ${ }^{2}$ The patients of CHD present as ACS a results of myocardial ischemia and its components are unstable angina (UA), non-ST-segment elevation myocardial infarction (NSTEMI), and ST-segment elevation myocardial infarction (STEMI). All three categories have ischemic symptoms in common. STEMI is always with persistent ST segment elevation or a new left bundle branch block on electrocardiography (ECG), NSTEMI is with elevated cardiac troponin levels but no ST segment elevation and Unstable angina has symptoms of cardiac ischemia without elevated ST-segment or cardiac troponin levels. ${ }^{3}$ The common presenting complaint of ACS patients in emergency is chest pain, retrosternal pressure or heaviness which may radiate to left arm, jaw or neck. ${ }^{4}$

The worldwide chief risk factors investigated for coronary artery disease may be high blood pressure, tobacco smoking and alcohol consumption whereas the high blood pressure has been listed as number one. ${ }^{5}$ The discrete statistics data for recent Global Burden of Diseases (GBD), have shown the hypertension as the top most risk factor worldwide in causing 
ischemic heart disease and stroke, so contributing to the Burdon. ${ }^{6}$ The prevalence of coronary artery disease (CAD) is globally rising especially in South-Asians who are targeted at relatively younger age compared to the inhabitants of other areas of the world. ${ }^{7}$ This rising trend may be attributed to industrialization, urbanization and posing changes in daily life and is called epidemiological transition. One of the major components of this transition is age of inactivity and obesity, when decreasing physical activity leads to epidemics of diabetes, hypertension and dyslipidemia which are responsible for growing CVD incidence and its associated death rates. ${ }^{8}$

Acute myocardial infarction is one of the top ten diseases which lead to increased mortality in elderly due to challenges like atypical symptoms, few changes on ECG and no significant levels of biochemical markers. These challenges pose difficulty in diagnosing AMI by treating physicians in elder patients. ${ }^{9}$ It has been found that STEMI type of AMI is globally the most important cause of death and morbidity. ${ }^{10}$

\section{MATERIAL \& METHODS}

This descriptive cross sectional study was carried out at the emergency department (ED) of Punjab institute of Cardiology (PIC) Lahore from November 2017 to January 2018. The study was approved by the ethical committee of the Postgraduate Graduate Medical Institute Lahore and was conducted with the approval of the head of PIC Lahore. A total of 170 patients with symptoms of ACS presenting to the ED and having final diagnosis of ACS were included in the study. The diagnosis was confirmed by the expert team of doctors working in emergency department. The inclusion criteria were patients of both sexes with age $\geq 35$ years, presenting with chest pain and/or dyspnoea within approximately 4 hours of onset and final diagnosis of ACS. The exclusion criteria were subjects who had undergone any cardiac intervention or thrombolysis at early stage and those with serum creatinine level $\geq 1.3 \mathrm{mg} / \mathrm{d}$. Informed written consent was taken from all the individuals included in the study.

At presentation history of presenting complaints,
ECG and Blood samples for Trop-T and routine investigations were also obtained from all patients. A next blood sample was drawn at $8 \mathrm{hrs}$ of arrival for the assessment of Troponin $T$ to confirm NSTEMI. General physical and systemic examination along with complete history including hypertension, smoking, diabetes and previous family history of ischemic heart disease was taken from all patients. The values of cardiac biomarkers (Troponin-T, CK-MB and CPK), creatinine, lipid profile and other routine investigations were also documented in the Performa. AMI was defined by characteristic clinical symptoms along with typical ECG (for STEMI) changes or cardiac necrosis marker cTnT (for NSTEMI) whereas UA was diagnosed if only typical symptoms were present and patient recovered with ACS Protocol. Data was analyzed statistically by SPSS software, version 18 and a P-value $\leq 0.05$ was considered significant.

\section{RESULTS}

One hundred and seventy patients; 139 (81.76\%) male and 31 (18.24\%) female of ACS based on clinical features, ECG changes and cardiac troponin $\mathrm{T}$ level, participated in the study. The mean age of the patients was 56 years while range of age was 35-85 years. Previous history of hypertension and IHD was present in 93 (54.71\%) and $64(37.65 \%)$ patients respectively while a family history of IHD was present in 64 (37.65\%) patients. Among 170 patients of ACS 61 (35.88\%) were smoker who were all male while 60 (35.29\%) were with hyperlipidemia and 55 (32.35\%) were known diabetic. Hyperlipidemia was defined if serum cholesterol, LDL and HDL cholesterol were deranged The socioeconomic status was also documented and the majority of the patients belonged to middle class (54.12\%) and lower class $(41.18 \%)$ whereas a few $(4.71 \%)$ belonged to upper class.

In ACS patient the main presenting complaint was typical chest pain (90\%) \pm other associated symptoms followed by chest heaviness (4.12\%) and dyspneoa (4.70\%) \pm other associated symptoms. One patient presented with epigastric pain and sweating, while another with fainting 
and vomiting. In 170 patients 131 (77.05\%) had AMI and 39 (22.95\%) UA. Among AMI patients, 111 (84.73\%) included STEMI and 20 (15.27\%) NSTEMI. The Anterior wall MI variety was the most frequent diagnosis $(n=57,51.35 \%)$ followed by inferior wall Ml variety $(n=52$, $46.85 \%)$ and isolated lateral wall $\mathrm{Ml}(\mathrm{n}=2,1.8$ $\%)$. The Anterior wall MI variety most frequently involved Anteroseptal MI (26.13\%) while among inferior wall $\mathrm{Ml}$ variety, the isolated inferior wall MI was most frequent (25.23\%) diagnosis. All the patients were managed on ACS protocol and three patients (1.77\%) expired and their diagnosis was STEMI; two during acute management due to cardiogenic shock and one due to cardiac arrest just before discharge from hospital.

\begin{tabular}{|l|c|}
\hline Anterior wall MI Variety & $\mathbf{5 7}(\mathbf{5 1 . 3 5 \% )}$ \\
\hline Anteroseptal MI & $29(26.13 \%)$ \\
\hline Extensive Anterior wall MI & $08(7.21 \%)$ \\
\hline Anterior wall MI & $06(5.41 \%)$ \\
\hline Anterolateral wall MI & $05(4.50 \%)$ \\
\hline Extensive anterolateral wall MI & $04(3.6 \%)$ \\
\hline Extensive anteroposterio wall \&RV MI & $01(0.90 \%)$ \\
\hline Anterior wall I \&R MI & $01(0.90 \%)$ \\
\hline $\begin{array}{l}\text { Anteroseptal MI \& Rt. bundle branch } \\
\text { block }\end{array}$ & $01(0.90 \%)$ \\
\hline $\begin{array}{l}\text { Anteroseptal MI \& Lt. bundle branch } \\
\text { block }\end{array}$ & $01(0.90 \%)$ \\
\hline Antero inferior MI & $01(0.90 \%)$ \\
\hline Inferior wall MI Variety & $\mathbf{5 2}(\mathbf{4 6 . 8 5 \% )}$ \\
\hline Inferior wall.23 MI & $28(25.23 \%)$ \\
\hline Infero posterior wall MI & $10(9 \%)$ \\
\hline Inferior wall \& RV MI & $09(8.11 \%)$ \\
\hline Inferolateral wall & $02(1.8 \%)$ \\
\hline Infero postero lateral wall MI & $01(0.90 \%)$ \\
\hline Infero postero lateral wall MI\&RV INF & $01(0.90 \%)$ \\
\hline $\begin{array}{l}\text { Inferior wall MI \& Lt. bundle branch } \\
\text { block }\end{array}$ & $01(0.90 \%)$ \\
\hline Isolated Lateral wall MI & $\mathbf{0 2}(\mathbf{1 . 8 \% )}$ \\
\hline & $\mathbf{n}=111)$ \\
\hline
\end{tabular}

\section{DISCUSSION}

CVDs have been the key basis of health loss globally and the ischemic heart disease (IHD) is the foremost cause of this worldwide phenomena occurring almost in every part of world. ${ }^{11} \mathrm{IHD}$ may affect persons at any age but becomes noticeably more common with advancing age, roughly tripling with every decade of life. Males are common victims compared to females. ${ }^{12}$ The majority of South Asian countries, including Pakistan, Sri Lanka, Bangladesh, India, and Nepal, cover more than a quarter of the low and middle-income countries and are recognized to have a top risk of coronary heart diseases (CHD) as compared to the rest of the world. ${ }^{13}$ Preventive strategies of IHD are targeted on detection and treating the risk factors both at the population and at personal levels by applying primordial, primary, and secondary preventive measures. ${ }^{14}$

The CVDs may besignificantly reduced by effective preventive measures focused on contributing factors. The important measures may include sufficient body exercise, loosing body weight, controlling BP, healthy food, lowering cholesterol levels and quitting smoking. Medications and exercise have almost equal effects in mortality reduction. ${ }^{15}$

In present study $81.76 \%$ were male and $18.24 \%$ were female showing a male to female ratio of $4.5: 1$ which is similar to various studies on ACS that have shown a male predominance. Gopalakrishnan et al $(2018)^{16}$ recruited a total of 236 patients of ACS in their study for different characteristics and reported $77.97 \%$ and $22.03 \%$ male and female respectively, with a male to female ratio of $3: 1$. The mean age of patients in our study was 56yrs which shows close similarity with the other studies done on in South Asian ACS patients. Sharma et al $(2014)^{17}$ analyzed 1562 patients of ACS and the mean age of patients were calculated to be $54.71 \mathrm{yrs}$ and the male were in majority (79.5\%). The frequent age of presentation of ACS was between 40-60years $(65.88 \%)$ in our study.

Hypertension appeared to be the most prominent modifiable risk factor (54.71\%) in our study and is consistent with the other studies. A study done in India by Mohanan et al $(2013)^{18}$ reported $48.8 \%$ hypertension in ACS patients while in another study done recently in China by Ge et al (2018) ${ }^{19}$ have documented $57.2 \%$ patients of ACS with hypertension. Our study reported that both history of previous IHD and family history (37.65\%) were 
same among all patient and these findings are almost in agreement with those of Ralapanawa et al $(2019)^{20}$ who carried out research on 300 ACS patients and investigated that previous history of IHD in patients and family history of IHD were $41 \%$ and $33 \%$ respectively. Our study has revealed the presence of hypercholesterolemia and diabetes mellitus in ACS cases as $35.29 \%$ and 32.35 respectively. These statistics are almost similar to work shown by Savith et al (2014) ${ }^{21}$ who found hypercholesterolemia and diabetes mellitus 30\% and $28 \%$ to be associated with ACS respectively. Our findings are also very much consistent with data presented by Farman et al $(2011)^{22}$ who calculated diabetes, smoking and hypertension, $38.9 \%, 24.8 \%$, and $47.8 \%$ respectively, in 113 ACS subjects. Contrary to our findings, a recent cross sectional study done by Adam et al (2017) ${ }^{23}$ on ACS patients in an urban tertiary care hospital in Pakistan has shown a maximum association of dyslipidemia (91.2\%), followed by hypertension $(70.4 \%)$ and diabetes $(51.2 \%)$. They found a family history of ACS (40.0\%) and smoking $(29.2 \%)$ which almost match with our results $(37.65 \%)$ and $(35.88 \%)$ respectively. They also calculated that $98.4 \%$ of patients were having at least one risk factor which matches with our (95.88\%) findings.

Most of our patients (90\%) had the typical chest pain at presentation and (10\%) atypical symptoms. Our findings are similar to those demonstrated King-Shier et al (2018) ${ }^{24}$ demonstrated approximately the similar results in South Asian ACS Patients; 81\% and 19\% typical and atypical symptoms respectively. STEMI has become a chief public health concern and in the present study it comprised $65.30 \%$, of ACS patients while remainder $22.95 \%$ and $11.77 \%$ with UA and NSTEMI respectively. Our results almost resemble with the results given by other studies done in Indo-Pak. Data published by study on ACS by Sharma et al (2014) ${ }^{17}$ revealed $63.7 \%, 25 \%$ and $11.3 \%$ STEMI UA and NSTEMI respectively.

In the present study among STEMI the most frequent variety was AWMI (51.35\%), followed by IWMI variety (46.85\%), and isolated LWMI (1.85\%).
Our findings are quite similar to those published by Zeb et al $(2017)^{25}$ which included IWMI variety (52.88\%), IWMI variety (44.01) and isolated LWMI (3.05\%). In-hospital death record in our ACS patients was $1.77 \%$ which is in accordance with the records published by Jafary et al $(2007)^{26}$ who noted an in hospital mortality in $2.5 \%$ of patients of ACS. Also our percentage of in-hospital expiry of patients is less than many previous and recent studies which is most probably due to early presentation as we only took those patients who presented within $4 \mathrm{hrs}$ of symptoms.

\section{CONCLUSION}

The profile of ACS patients shows that a majority of ACS patients in our country is male and the most prominent diagnosis is STEMI of which anterior wall $\mathrm{Ml}$ variety is predominant. The major modifiable risk factors are hypertension, smoking, hyperlipidemia and diabetes $90 \%$ patients present with typical chest pain while remaining $10 \%$ atypical symptoms which must be of prime consideration to assess ACS. Education of public in medical office setting and in the community is important because timely treatment of risk factors can reduce the burden of IHD. Moreover, the minimal delay on the patient's side to seek medical help as well as careful assessment from local physicians is also recommended.

Copyright $@ 21$ Feb, 2020.

\section{REFERENCES}

1. World Health Organization. Global health estimates 2016: disease burden by cause, age, sex, by country and by region, 2000-2016. Geneva: World Health Organization. 2018.

2. Yusuf S, Rangarajan S, Teo K, Islam S, Li W, Liu L, Bo J, Lou Q, Lu F, Liu T, Yu L. Cardiovascular risk and events in 17 low-, middle-, and high-income countries. New England Journal of Medicine. 2014 Aug 28;371(9):818827.

3. O'Gara PT, Kushner FG, Ascheim DD, Casey DE, Chung MK, De Lemos JA, Ettinger SM, Fang JC, Fesmire FM, Franklin BA, Granger CB. 2013 ACCF/AHA guideline for the management of ST-elevation myocardial infarction: a report of the American College of Cardiology Foundation/American Heart Association Task Force on Practice Guidelines. Journal of the American College of Cardiology. 2013 Jan 29;61(4):e78140. 
4. Steg G, James SK, Atar D, Badano LP, BlomstromLundqvist $\mathrm{C}$, Borger MA, et al. ESC Guidelines for the management of acute myocardial infarction in patients presenting with ST-segment elevation. Eur Heart J.2012; 33(20):2569-2619.

5. Lim SS, Vos T, Flaxman AD, Danaei G, Shibuya K, AdairRohani $\mathrm{H}$, et al. A comparative risk assessment of burden of disease and injury attributable to 67 risk factors and risk factor clusters in 21 regions, 19902010: a systematic analysis for the Global Burden of Disease Study 2010. The Lancet 2012; 380(9859):22242260.

6. GBD (Global Burden of Diseases) 2016 Risk Factors Collaborators. Global, regional, and national comparative risk assessment of 84 behavioral, environmental and occupational, and metabolic risks or clusters of risks, 1990-2016: a systematic analysis for the Global Burden of Disease Study 2016. Lancet 2017; 390(10100):1345-1422.

7. Volgman AS, Palaniappan LS, Aggarwal NT, Gupta $M$, Khandelwal $A$, Krishnan $A V$, et al; on behalf of the American Heart Association Council on Epidemiology and Prevention; Cardiovascular Disease in Women and Stroke and Special Populations Committee of the Council on Clinical Cardiology; Council on Cardiovascular and Stroke Nursing; Council on Quality of Care and Outcomes Research; and Stroke Council. Atherosclerotic cardiovascular disease in South Asians in the United States: epidemiology, risk factors, and treatments: a scientific statement from the American Heart Association. Circulation. 2018; 138:e1e34.

8. Gaziano TA, Gaziano JM. Epidemiology of cardiovascular disease. Fauci AS, Braunwald E, Kasper DL, Hauser SL, Longo DL, Jameson JL, et al. Harrison Principals of Internal Medicine. 17th ed. Mc-Graw Hills. 2008:1377-78.

9. Ambali AP, Bomman JV. Clinical profile of $\mathbf{1 5 0}$ elderly patients with acute myocardial infarction. J. Evid. Based Med. Healthc. 2018; 5(17), 1435-1438.

10. Ahmed N, Carberry J, Teng V, Carrick D, Berry C. Risk assessment in patients with an acute ST-elevation myocardial infarction. J Comp Eff Res. 2016; 5(6):581593.

11. Roth GA, Johnson C, Abajobir A, Abd-Allah F, Abera SF, Abyu G, et al. Global, Regional, and National Burden of Cardiovascular Diseases for 10 Causes, 1990 to 2015. J Am Coll Cardiol. 2017; 70(1):1-25.

12. Finegold JA, Asaria P, Francis DP. Mortality from ischaemic heart disease by country, region, and age: Statistics from World Health Organisation and United Nations. Int J Cardiol. 2013; 30; 168(2):934-945.
13. WHO. Global status report on non-communicable diseases 2014. Media Centre 2017. [cited 2016 May 1]. Available from: URL: http://www.who.int/nmh/ publications/ncd-status-report-2014/en/.

14. Wong, ND. Epidemiological studies of CHD and the evolution of preventive cardiology. Nat Rev Cardiol. 2014; 11(5):276-289.

15. Naci H, loannidis JP. Comparative effectiveness of exercise and drug interventions on mortality outcomes: Metaepidemiological study. BMJ. 2013 Oct $1 ; 347:$ :5577.

16. Gopalakrishnan S and Govindharaju A. Clinical, ECG and echocardiographic profile of patients presenting with acute St Elevation Myocardial Infarction (STEMI) in a tertiary care institute at Tamilnadu, South India. J Evid Based Med Healthc. 2018; 5(45):3131-3136.

17. Sharma R, Bhairappa S, Prasad SR and Manjunath CN. Clinical characteristics, angiographic profile and in hospital mortality in acute coronary syndrome patients in south Indian population. Heart India. $2014 ; 2$ (3):65-69.

18. Mohanan PP, Mathew R, Harikrishnan S, Krishnan MN, Zachariah G, Joseph J, et al. Presentation, management, and outcomes of 25748 acute coronary syndrome admissions in Kerala, India: Results from the Kerala ACS registry. Eur Heart J. 2013; 34(2):121129.

19. Ge J, Li J, Yu H, Hou B. Hypertension Is an Independent Predictor of Multivessel Coronary Artery Disease in Young Adults with Acute Coronary Syndrome. International Journal of Hypertension. 2018 Nov 13;2018.

20. Ralapanawa U, Kumarasiri PV, Jayawickreme KP, Kumarihamy $P$, Wijeratne $Y$, Ekanayake $M$, et al. Epidemiology and risk factors of patients with types of acute coronary syndrome presenting to a Tertiary Care Hospital in Sri Lanka. BMC Cardiovasc Disord. $2019 ; 19(1): 229$.

21. Savith A. Clinical profile of acute myocardial infarction in elderly patients: A cross sectional study. Int J Sci Study. 2015; 3(6):65-68.

22. Farman MT, Sial JA, Khan NU, Rizvi SN, Saghir T, Zaman KS. Outcome of primary percutaneous coronary intervention at public sector tertiary care hospital in Pakistan. JPMA-Journal of the Pakistan Medical Association. 2011 Jun 1; 61(6):575. 
23. Adam AM, Rehan A, Waseem N, lqbal U, Saleem $H$, Ali MA, et al. Prevalence of conventional risk factors and evaluation of baseline indices among young and elderly patients with coronary artery disease. J Clin Diagn Res. 2017; 11(7):OC34-OC39.

24. King-Shier K, Quan H, Kapral MK, Tsuyuki R, An $\mathrm{L}$, Banerjee $\mathrm{S}$, et al. Acute coronary syndromes presentations and care outcomes in white, South Asian and Chinese patients: A cohort study. BMJ Open 2019; 9:e022479. doi: 10.1136/bmjopen-2018-022479.
25. Zeb S, Hafizullah M, Jan H, Adil M. and Zeb R. ST elevation myocardial infarction: An experience at Lady Reading Hospital Peshawar, Pakistan. Khyber Med Univ J. 2017; 9(2): 60-62.

26. Jafary $\mathrm{MH}$, Samad A, Ishaq M, Javaid SA, Mansoor A and Vohra EA. Profile of Acute Myocardial Infarction (AMI) in Pakistan. PAK J MED SCI. 2007; 23(4):485489.

\begin{tabular}{|c|c|c|c|}
\hline \multicolumn{4}{|c|}{ AUTHORSHIP AND CONTRIBUTION DECLARATION } \\
\hline Sr. \# & Author(s) Full Name & Contribution to the paper & Author(s) Signature \\
\hline 1 & Zahid Mahmood & $\begin{array}{l}\text { Conception, Design, Synthesis } \\
\text { and planning of the research, } \\
\text { Active participation idea data } \\
\text { collection and management, } \\
\text { Drafting of article. }\end{array}$ & \\
\hline 2 & Tariq Feroze Khawaja & $\begin{array}{l}\text { Participation in active } \\
\text { methodology, Assembly of } \\
\text { data, Statistical expertise. }\end{array}$ & \\
\hline 4 & Anjum lqbal & Critical revision of the article for & \\
\hline 5 & Abdul Rasnid Knan & $\begin{array}{l}\text { Analysis and interpretation of } \\
\text { the data. }\end{array}$ & \\
\hline & Naveed Arshad & $\begin{array}{l}\text { Participation in Statistical } \\
\text { analysis and drafting. }\end{array}$ & \\
\hline
\end{tabular}

